\title{
Dynamic Control of Contractile Force in Engineered Heart Tissue
}

This paper was downloaded from TechRxiv (https://www.techrxiv.org).

\section{LICENSE}

CC BY 4.0

SUBMISSION DATE / POSTED DATE

20-07-2021 / 25-07-2021

\section{CITATION}

Bifano, Thomas; Chen, Chris; Li, Huate; Sundaram, Subramanian; lou, lihua; agarwal, arvind; et al. (2021): Dynamic Control of Contractile Force in Engineered Heart Tissue. TechRxiv. Preprint. https://doi.org/10.36227/techrxiv.15025236.v1

$\mathrm{DOI}$

10.36227/techrxiv.15025236.v1 


\title{
Dynamic Control of Contractile Force in Engineered Heart Tissue
}

\author{
Huate Li, Subramanian Sundaram, Ruifeng Hu, Lihua Lou, Arvind Agarwal, Christopher S. Chen, and \\ Thomas G. Bifano*1
}

\begin{abstract}
Three-dimensional engineered heart tissues (EHTs) derived from human induced pluripotent stem cells (iPSCs) have become an important resource for both drug toxicity screening and research on heart disease. A key metric of EHT phenotype is the contractile force with which the tissue spontaneously beats. It is well-known that cardiac muscle contractility - its ability to do mechanical work - depends on tissue prestrain (preload) and external resistance (afterload). Objectives: Here, we demonstrate a technique to control both preload and afterload dynamically while monitoring contractile force exerted by EHTs. Methods: We developed an apparatus that uses real-time feedback control to monitor and regulate EHT forces. The system is comprised of a pair of high-speed piezoelectric actuators that can strain the EHT scaffold and a fast optical measurement tool to provide EHT contractile force feedback while monitoring tissue strain. Results: The system was used to regulate the effective stiffness of the scaffold. When controlled to have effectively isometric boundary conditions, EHTs exerted a contractile force that was almost twice as large as that observed under auxotonic conditions. Conclusion: These experimental results demonstrate that EHT contractility can be increased through feedback control to regulate boundary stiffness. Significance: The work advances our understanding of the role that mechanical environment plays in EHT contractility. This could be used to help study or alter EHT phenotype and potentially EHT maturation through controlled mechanical conditioning.
\end{abstract}

Index Terms - Biomedical Imaging, Cardiomyocyte, Contractility, Force Control, Tissue Engineering

\section{INTRODUCTION}

E NGINEERED heart tissues (EHTs) derived from human induced pluripotent stem cells (iPSCs) are used as in vitro models to study cardiac physiology [1-3]. One such engineered structure is the microfabricated tissue gauge ( $\mu \mathrm{Tug}$ ), an elastomer scaffold comprised of a microwell with a pair of vertical compliant pillars extending upward from its base. The tops of the pillars serve as anchors for self-assembly of 3D EHTs that

${ }^{1}$ Submitted July 30, 2021. This work was supported in part by the Engineering Research Centers Program of the National Science Foundation under NSF cooperative agreement no. EEC-1647837. S.S. is supported by American Heart Association grant 20POST35210045.

$\mathrm{H}$. Li is with the Mechanical Engineering Department, Boston University, Boston, MA 02215 USA (huateli@bu.edu).

S. Subramanian is with the Biomedical Engineering Department, Boston University, Boston, MA 02215 USA (subras@bu.edu).

$\mathrm{R}$. Hu is with the Mechanical Engineering Department, Boston University, Boston, MA 02215 USA (ruh24@bu.edu). form over a period of days from a cell-laden gel of natural extracellular matrix materials [4]. An attribute of the cardiac $\mu$ Tug is that these tissue constructs made of iPSC-derived cardiomyocytes and supporting cells - such as Fibroblasts or human mesenchymal stem cells (hMSCs) - form spontaneously beating $3 \mathrm{D}$ structures that recapitulate some of the important physiology of cardiac muscle. In addition to $\mu$ Tugs, 3D EHTs can be made using approaches that include cells molded in hydrogels and self-assembled tissues formed around biowire scaffolds. These approaches also allow suspension of the 3D EHT and optical monitoring of tissue contractile tension $[5,6]$. When $\mu$ Tug EHTs are fully compacted, they begin periodic spontaneous contraction. The resulting tensile force bends the pillar tops measurably toward one another. With a suitable imaging system and knowledge of pillar mechanical properties, one can infer EHT contractile force resulting from spontaneous contraction, which is a key functional metric for evaluating the performance of cardiac microtissues.

Contraction dynamics of EHTs derived from iPSCs have been studied widely, and are characterized by parameters such as peak tension, peak rate of change of tension for contraction and relaxation, and time from stimulus to peak tension [7]. These are sometimes used as surrogate measures of tissue maturation. A profound functional difference between EHTs derived from iPSCs and adult human cardiac muscle is the substantially larger contractility (ability to do mechanical work) of the latter.

In $\mu$ Tug scaffolds, the stiffness of the pillars impacts the magnitude of force generated by EHTs; stiffer pillars have been shown to increase contractile force and tension [8]. Compliant scaffolds for EHTs can be modified mechanically to precondition the EHT using initial stretching (preload), or to alter its external resistance (afterload) [9, 10]. Preconditioning has been shown to increase EHT maturity and to increase EHT peak contractile force.

Modulation of substrate stiffness is known to be essential for

L Lou is with the Department of Mechanical and Materials Engineering, Florida International University, Miami FL 33174, USA (llou@fiu.edu).

A. Agarwal is with the Department of Mechanical and Materials Engineering, Florida International University, Miami FL 33174 USA (agarwala@fiu.edu).

C. S. Chen is with the Biomedical Engineering Department, Boston University, Boston, MA 02215 USA (chencs@bu.edu).

* Corresponding Author T. G. Bifano is with the Mechanical Engineering Department, Boston University, Boston, MA 02215 USA (tgb@bu.edu). 
the process of maturation in vivo. Driven by the accumulation of extracellular matrix materials like collagen, the ECM stiffness increases several fold from embryonic to the adult stage [11]. Motivated by this, there are numerous previously reported studies of scaffolds that allow some dynamic mechanical control of EHTs, using electromagnetic, pneumatic, or piezoelectric [12-15] actuation. A few studies also incorporate dynamic force control to regulate and measure tissue tension simultaneously [16-18].

In this work we aim to improve our quantitative understanding of the effect of afterload on tissue contractility by monitoring the contractile forces exerted by EHTs in a scaffold that can be controlled dynamically to alter its effective stiffness during testing. The extremes for such mechanical scaffold constraints in $\mu$ Tugs are isometric (perfectly rigid pillars) and isotonic (perfectly compliant pillars). Real pillars provide auxotonic boundary conditions that fall somewhere in between these extremes.

In the work described here, we incorporate a real-time force feedback control system with a $\mu$ Tug to allow comparison of various preload and afterload effects on contractile forces in a single EHT. The system can measure and control dynamic forces on engineered heart tissues (EHTs) in real time. The effective stiffness of the scaffold is maintained artificially through closed-loop control of a pair of mechanically amplified piezoelectric actuators, each comprised of a stack of piezoceramic transducers that bend a flexural steel housing when energized by an external voltage, permitting dynamic linear translation of up to $1.5 \mathrm{~mm}$ per actuator. These actuators are mounted on opposing sides of the $\mu$ Tug scaffold and are used to stretch or compress it rapidly in response to measured force and contraction dynamics. The system employs a highspeed CMOS camera integrated with an inverted widefield microscope to monitor pillar deformation, scaffold strain, and EHT strain, from which EHT contractile force (or tension) can be inferred. A simplified schematic of the system is depicted in Figure 1.

The system is capable of subjecting EHTs to boundary constraints that range from effectively isometric to isotonic. It can switch in milliseconds between those conditions, and it can apply dynamic EHT strains measuring up to $13 \%$ in the central region of the EHT. This dynamic strain is additive to a preload strain that can be applied to the EHT by statically stretching the scaffold by up to $50 \%$ through adjustment of a mechanical slideway. An image of the molded polydimethylsiloxane (PDMS) $\mu$ Tug scaffold adapted for this system is shown in Figure 2.

System operation for isometric control, for example, is illustrated schematically in Figure 3. In this case, the control objective is to maintain the pillar tops a fixed distance apart, counteracting deformation that would otherwise occur due to EHT contractile forces.
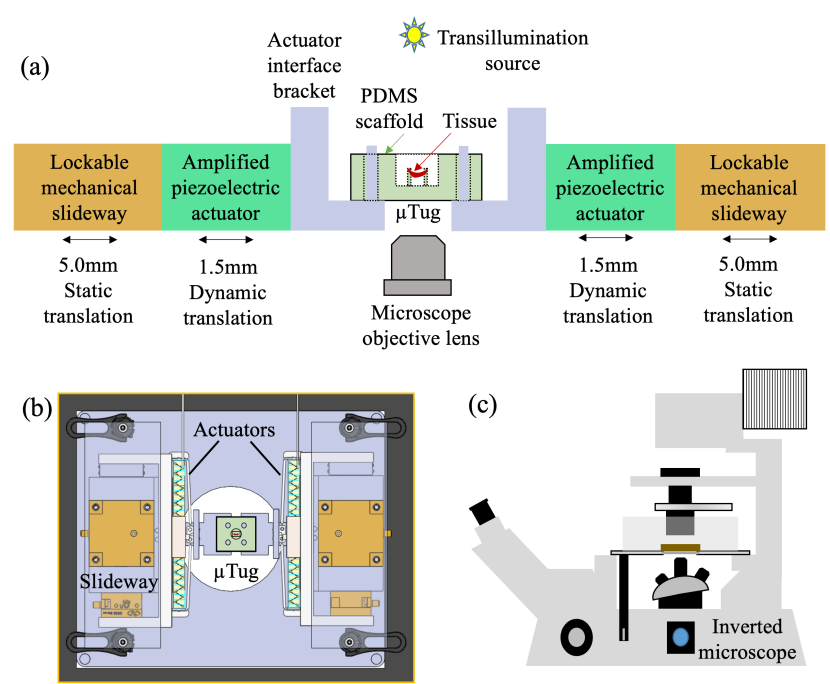

Fig. 1. Schematic of the dynamic force control system. (a) Cross sectional schematic depiction of the main actuation and measurement components. The $\mu$ Tug scaffold with PDMS pillars in a media-filled well containing the EHT is mounted on protruding prongs of an actuator mounting bracket. The bracket is fixed to one end of an amplified piezoelectric actuator that can impose dynamic tensile strain on the $\mu$ Tug scaffold. The other end of the actuator is attached to a lockable mechanical slideway that can be adjusted to adjust static tensile strain of the $\mu$ Tug scaffold. A transilluminated microscope objective focuses on the scaffold pillar tops and well edges to monitor tissue force and strain. (b) Top view CAD schematic of the actuator and slideway assembly with a $\mu$ Tug. This assembly is maintained in an environmental chamber that regulates temperature, humidity, and $\mathrm{CO}_{2}$. (c) Side view schematic of the inverted microscope upon which the force control system is mounted.

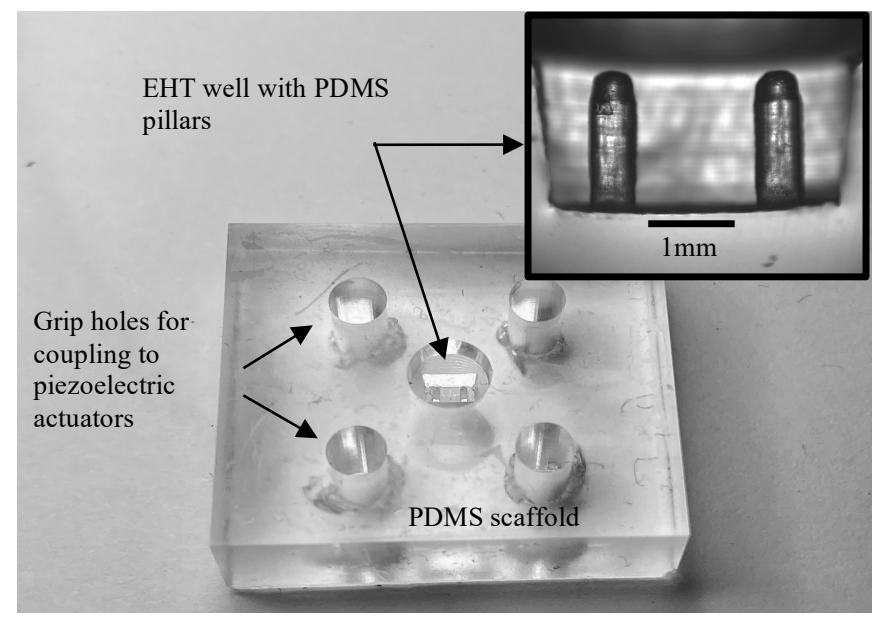

Fig. 2. Photo of the PDMS scaffold, fabricated using direct molding from a $3 D$ printed template. The structure measures $5 \mathrm{~mm}$ tall, $25 \mathrm{~mm}$ deep, and 20 $\mathrm{mm}$ wide. It features two pairs of through holes spaced $10 \mathrm{~mm}$ apart to the left and right of the EHT well. Each pair of holes aligns with prongs on an actuator interface bracket that is rigidly attached to an amplified-displacement piezoelectric transducer. The piezoelectric actuators can stretch the scaffold by up to $3 \mathrm{~mm}$ via high-voltage analog input. Inset: Side view microscope image of PDMS pillars. 
(a)

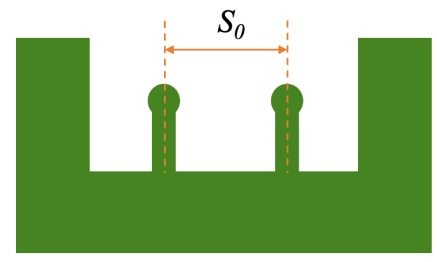

(b)

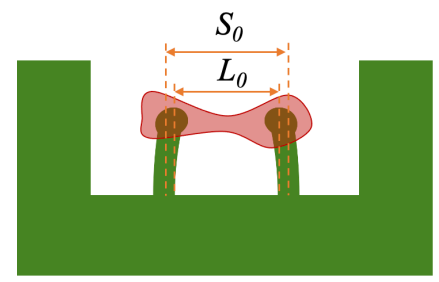

(c)
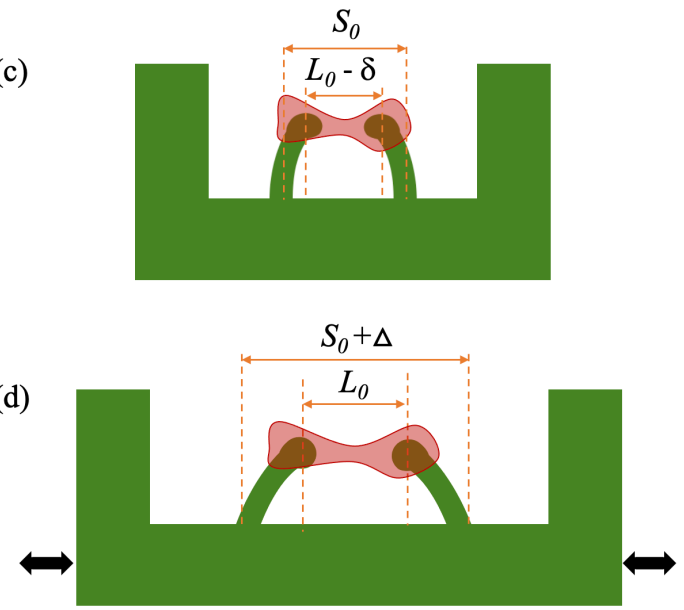

Fig. 3. Cross sectional schematic of the $\mu$ Tug pillars and tissue well to illustrate basic system function for isometric control. (a) $\mu$ Tug system is comprised of a PDMS scaffold and a pair of PDMS pillars in a well. Prior to EHT formation, scaffold pillar bottoms are separated laterally by an amount $S_{0}$. (b) Cardiomyocytes derived from iPSCs form a compacted EHT spanning the pillars. Static tension exerted by the EHT deflects the pillar tops toward one another. The initial length of the EHT is $\boldsymbol{L}_{\boldsymbol{0}}$. (c) Without control, spontaneous periodic EHT contraction deflects pillar tops toward one another by an amount $\delta$. Assuming linear elastic pillars, EHT tensile force magnitude can be estimated as $\boldsymbol{k}_{\boldsymbol{p}} \boldsymbol{\delta} / \mathbf{2}$, where $\boldsymbol{k}_{\boldsymbol{p}}$ is the pillars' combined bending and shear stiffness. (d) During isometric control, the actuators are energized to stretch the scaffold by an amount $\boldsymbol{\Delta}$ in response to measured pillar deformation, with a control objective to keep pillar tops always separated by a distance $\boldsymbol{L}_{\boldsymbol{0}}$, counteracting any change in EHT length in response to a spontaneous contractile force. Controller bandwidth and range is sufficient to effectively suppress pillar spontaneous contraction dynamics.

\section{MAterials AND MethodS}

\section{A. Elastomeric Scaffold and EHT Preparation}

The molds for generating the elastomeric scaffolds were designed using CAD tools (Solidworks) and 3D-printed with the MicroFine ${ }^{\mathrm{TM}}$ Green resin by Stereolithography (Protolabs, Maple Plain, MN, USA). The printed parts received were rinsed in ethanol and then blown dry with an air gun. The molds were subsequently treated in $100 \mathrm{~W}$ air plasma for $30 \mathrm{~s}$ and then treated with trichloro $(1 \mathrm{H}, 1 \mathrm{H}, 2 \mathrm{H}, 2 \mathrm{H}$-perfluorooctyl)silane under low vacuum overnight. After silane treatments, all PDMS scaffolds were made using these molds with a 1:20 mixture of crosslinker to monomer (Sylgard 184 Silicone Elastomer Kit), and cured overnight at $60^{\circ} \mathrm{C}$. After demolding, the PDMS parts were further cured overnight at $100^{\circ} \mathrm{C}$ in a convection oven.

Prior to cell seeding, the PDMS scaffolds were plasma treated for 30 s followed by a $2 \mathrm{~h}$ treatment in $0.01 \%$ poly-Llysine (PLL) and 1\% glutaraldehyde (Electron Microscopy Sciences, Inc) for 10min. These treatments help the EHT adhere to the top caps of the PDMS devices. The scaffolds were rinsed thrice in PBS and let to sit at $4^{\circ} \mathrm{C}$ for two nights in DI water. The PDMS scaffolds were then soaked in $100 \%$ ethanol for $15 \mathrm{~min}$, dried and UV sterilized for $15 \mathrm{~min}$. The devices were transferred to a tissue culture hood and the bottom portion of the rectangular well was treated with $2 \%$ Pluronic F-127 for 30min to prevent adhesion of the EHT to the bottom surface of the rectangular well.

The cardiomyocytes used here under Boston University IRB Protocol 1820, approved February 2020, were generated from iPSCs using the PGP1 parent line (white adult male, born 1954), and differentiated by temporal modulation of regulators of the Wnt signaling pathway [19]. After differentiation, the hiPSC-derived CMs were purified by lactate starvation for 4 days (glucose free RPMI with $4 \mathrm{mM}$ sodium lactate). Once purified, the iPSC-CMs were transferred to fresh Fibronectin treated plates and maintained in RPMI containing B-27 Supplement (1:50 dilution) for up to a month. A mixture of $90 \%$ iPSC-CMs and 10\% hMSCs totaling 60,000 cells and suspended in $5.5 \mathrm{uL}$ of extracellular matrix (ECM) mixture containing $4 \mathrm{mg} / \mathrm{ml}$ Fibrin (human Fibrinogen, MilliporeSigma with $0.4 \mathrm{U} / \mathrm{mg}$ Thrombin, MilliporeSigma) and 10\% Matrigel (Corning) was added to each PDMS well. The PDMS scaffolds were placed upside down for the cells to settle close to the top of the pillars during polymerization.

After crosslinking $(\sim 5 \mathrm{~min})$, the cell-laden hydrogel was cultured in growth media made with high-glucose DMEM containing $10 \%$ fetal bovine serum (MilliporeSigma), $1 \%$ penicillin-streptomycin, 1\% nonessential amino acids (Fisher) and $1 \%$ GlutaMAX (Fisher) supplemented with 5 $\mu \mathrm{M}$ Y-27632 and $33 \mu \mathrm{g} / \mathrm{ml}$ aprotinin. About $50 \mu \mathrm{L}$ of growth media was added to each PDMS device containing the cell-laden hydrogel and stored in an incubator at $37^{\circ} \mathrm{C}$ with $5 \% \mathrm{CO}_{2}$. The ROCK inhibitor Y-27632 was removed after 1 day of culture and the media was replaced every two days. Over the course of 2-5 days, the cell-laden ECM gradually compacts to form the EHT.

\section{B. $\mu$ Tug Microscope Force Control System}

The PDMS (Sylgard-184, Dow) scaffold contains a central well region that contains the self-assembled tissue and tissue culture media during testing. Pillars are rectangular in cross section, measuring $450 \mu \mathrm{m}$ in diameter and $1450 \mu \mathrm{m}$ tall. Pillar separation is $1580 \mu \mathrm{m}$. Pillar lateral stiffness (i.e., combined bending and shear stiffness) is measured using an in-situ indenter for soft biomaterials (Hysitron Biosoft, Bruker).

The $\mu$ Tug scaffold is mounted to an actuator interface bracket by aligning holes in the scaffold with prongs protruding from the backet, as depicted in Figs.1 and 2. The other side of 
the bracket is bolted to one side of a mechanically amplified piezoelectric actuator (APF710, Thorlabs). The opposing side of the actuator is mounted to a lockable mechanical slideway that is mounted to a rigid microscope base plate. The mechanical slideway can be adjusted to impose a nominal static strain to the scaffold ranging from $0-50 \%$. The actuators are controlled using a data acquisition module (PCI6251, National Instruments) and a multichannel high voltage piezoelectric actuator driver (MDT693B, Thorlabs). The piezoelectric actuators have a resonant frequency of about $185 \mathrm{~Hz}$. The pair of actuators combine to allow a full range of motion of $3 \mathrm{~mm}$ with a resolution of $\sim 50 \mathrm{~nm}$ and can provide $0-13 \%$ dynamic strain to the scaffold that is additive to any initial static scaffold strain imposed by the lockable mechanical slideway. The base plate features a window at its center to allow optical access for an inverted microscope that is focused on the EHT. The transilluminated microscope (IX73, Olympus) uses a $4 x$ (0.16NA) objective lens (UPLFLN4X, Olympus) and a fast CMOS camera (PCO Edge4.2LT, PCO AG) along with a $2 x$ reducing telescope to image the EHT in real time. The microscope resolution is $\sim 3 \mu \mathrm{m}$, and the camera resolution is matched to this with $\sim 3 \mu \mathrm{m} /$ pixel. The imaging field of view can be as large as $6 \mathrm{~mm} \times 6 \mathrm{~mm}$ for initial setup and alignment. To allow faster acquisition during control, the field of view is truncated to $6 \mathrm{~mm} \times 48 \mu \mathrm{m}$ and oriented so that the centers of the pillar tops and the well edges are included in the frame. A custom-built side-view microscope is used to image pillars and EHT through the side of PDMS scaffold prior to control experiments. This image is used to determine initial tissue pillar bending and to provide a sectional view of EHT geometry and its attachment to pillars.

The base plate, actuators, and scaffold are enclosed in a custom-built environmental enclosure that regulates temperature, humidity, and carbon dioxide concentration at set points of $37^{\circ} \mathrm{C}, 50 \%$, and $5 \%$ respectively.

\section{Contractile Force Measurement}

Reference images of scaffolds without EHT and with no applied scaffold strain are used to establish baseline measurements of well width and pillar separation. Before control, the $\mu$ Tug with EHT is mounted to the actuator mounting plate and adjusted using the mechanical slideway to produce a prescribed static scaffold strain before being locked into place. The microscope is adjusted to center the image of pillars in the field of view and to focus on the pillar tops and well edges. An initial reference image of the pillars and well edges is acquired. A typical image is depicted in Figure 4.

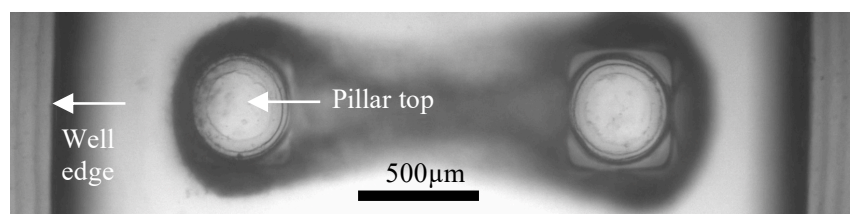

Fig. 4. Microscope image of $\mu$ Tug with EHT. Pillar tops and well edges are in sharp focus. Actuation is in the horizontal direction and produces uniaxial strain on the scaffold resulting in changes in tissue tension.

The closed-loop force control system cycle time is $\sim 3.2 \mathrm{~ms}$, corresponding to a $312 \mathrm{~Hz}$ image acquisition frame rate. In each cycle, the camera acquires an image of the pillars and the well edges. Four selected subregions of the control image corresponding to the pillar tops and the well edges are cross correlated with corresponding subregions on the reference image using an intrinsic MATLAB image cross correlation function to estimate scaffold well edge separation and pillar top separation with sub-micrometer localization precision. As expected, localization is more precise than nominal image resolution. The typical noise floor for localization, given the selected imaging system and image correlation algorithms, is $\sim 0.25 \mu \mathrm{m}$.

The two system variables that are readily measured from cross-correlated images are tissue length, $\boldsymbol{L}$, defined by the lateral separation of pillar tops, and scaffold strain, $\boldsymbol{\varepsilon}_{s}$ defined as the change in lateral separation of the $\mu$ Tug well edges divided by the initial (unstrained) separation of the well edges.

To estimate EHT force, we need to know pillar deformation, $\delta$, defined as the difference between the lateral separation of pillar bottoms and the lateral separation of pillar tops, $\boldsymbol{L}$. However, pillar bottoms are not in focus during control. Fortunately, there is a direct linear relationship between well edge separation (or, equivalently, measured scaffold strain $\boldsymbol{\varepsilon}_{s}$ ) and pillar bottom separation. Because the $\mu$ Tug scaffold base layer in which the pillars are fixed is orders of magnitude stiffer than the EHT, this relationship is not affected by the presence of tissue. In a pre-calibration step, we added a second camera to the imaging system that focuses on the well bottom. In this image, pillar bottoms were in sharp focus. Then we used the piezoelectric actuators to strain the scaffold while recording the well edge separation (via the first camera) and the pillar bottom separation (via the second camera). We repeated this calibration for every scaffold before seeding the EHT tissue. We used this calibration in all subsequent experiments to infer pillar bottom separation from measured well separation or scaffold strain. From this data, the instantaneous EHT force or tension can be calculated in real time as the measured lateral deformation of the pillars (i.e., the difference between pillar bottom separation and pillar top separation) multiplied by the lateral pillar stiffness. Lateral pillar stiffness was measured using an in-situ indenter after completion of control experiments. Tissues were first removed from the $\mu$ Tug, and the scaffolds were trimmed and mounted on glass slides to expose individual pillars to the indenter. The indentation test included first contacting the top of the pillar, then applying $100 \mu \mathrm{m}$ of lateral displacement at $5 \mu \mathrm{m} / \mathrm{s}$ loading rate, followed by unloading at the same rate. From the slope of the nominally linear unloading curve the stiffness of the pillars could be calculated directly. Based on the measured lateral stiffness of pillars and the noise floor of the cross-correlated localization measurements of pillar deflection, we estimate the EHT contractile force measurement noise floor to be $\sim 0.5 \mu \mathrm{N}$.

Changes in contractile force $\boldsymbol{F}_{\boldsymbol{p}}$ at the top of each pillar are determined uniquely by pillar deflection, $\delta$, and the lateral mechanical stiffness of the pillar, $\boldsymbol{k}_{\boldsymbol{p}}$. Since there are two 
pillars, the net boundary stiffness experienced by the tissue is $\boldsymbol{k}_{\boldsymbol{p}} / \mathbf{2}$ (without control). Consequently,

$$
F_{p}=\frac{\delta k_{p}}{2}
$$

Figure 5 is a plot of spontaneous contractile force as a function of time over a twelve second period for a typical EHT sample in a $\mu$ Tug, measured prior to control. The EHT contractile force in this case measured about $45 \mu \mathrm{N}$ peak-tovalley.

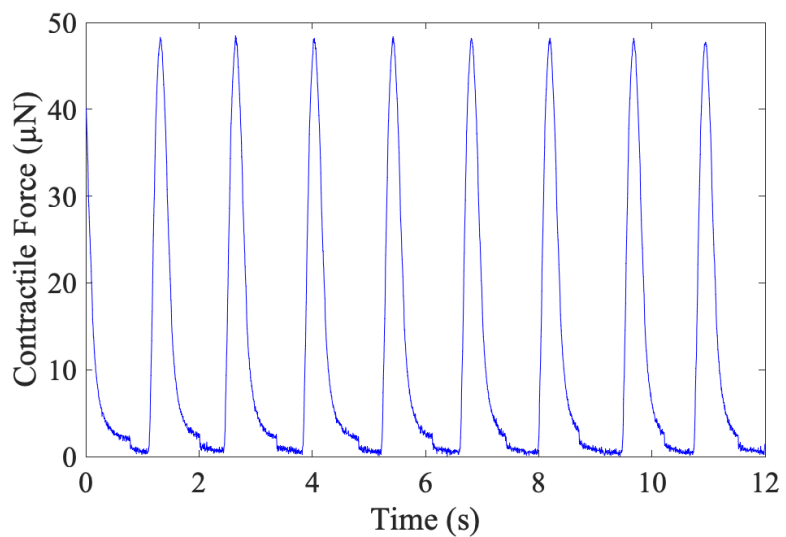

Fig. 5. Measured EHT contractile force with respect to nominal EHT tensile force, for a typical $\mu$ Tug sample. The pace of spontaneous contraction is $\sim 0.74 \mathrm{~Hz}$, and the mean contractile peak force is $\sim 45 \mu \mathrm{N}$.

The instantaneous effective stiffness, $\boldsymbol{k}_{\boldsymbol{e f f}}$, of the boundary attachments from the perspective of the EHT can be defined as the force on the pillars divided by the net change in length of the tissue:

$$
\boldsymbol{k}_{\text {eff }}=\frac{\boldsymbol{F}_{p}}{\left(\boldsymbol{L}-\boldsymbol{L}_{0}\right)}
$$

where $\boldsymbol{L}_{\mathbf{0}}$ is the reference length for the EHT measured when the scaffold actuators are not energized, and $\boldsymbol{L}$ is the instantaneously measured tissue length which is equivalent to the pillar top separation. Note that if the scaffold strain is held at zero (i.e., no actuation), then $\boldsymbol{\delta}=\boldsymbol{L}-\boldsymbol{L}_{\mathbf{0}}$ so $\boldsymbol{k}_{\text {eff }}=\boldsymbol{k}_{\boldsymbol{p}} / \mathbf{2}$, as expected. To make the effective stiffness as large as possible, the control objective is to maintain the condition $\boldsymbol{\delta}=\boldsymbol{0}$ : constant pillar top separation.

We measure forces and strains of the tissue relative to the reference state of the EHT at rest in the unstrained $\mu$ Tug (at length $\boldsymbol{L}_{\boldsymbol{0}}$ ). Of course, the internal strain of the suspended EHT is not zero in this reference state, and neither is its tensile force zero. Even in the relaxed state in an unstrained scaffold, the EHT exerts a tensile force on the pillars. For the EHT, zero internal strain and zero tensile force corresponds to the state that the EHT would be in if it were released from its boundary constraints. Empirically, we have observed (by releasing one end of the $\mu \mathrm{Tug}$ ) that in the zero-strain state the tissue nominal length is approximately $\mathrm{L}_{0} / 2$.

\section{Scaffold and EHT Strain Measurement}

EHT phenotype may be affected by tissue strain or preload. Consequently, it is important in the control experiments reported here to measure the nominal EHT strain during control. In the images used for feedback control, the pillar tops and tissue well edges are in the focus plane of the microscope, but the EHT is not in sharp focus. That prevents optical tracking of tissue strain in real time. However, we can measure scaffold strain directly during control, and we can map the EHT strain for any sample to that scaffold strain through a calibration process. For that calibration we impose a quasistatic open-loop ramp on the actuators to produce both scaffold strain and tissue strain. We focus the microscope main camera on the EHT layer and track the strain of tissue features in a central region of the EHT defined as a rectangle comprising the central third of the EHT span between pillars and one half of its minimum width. We also track the strain of the scaffold by measuring separation of the well-edges. Figure 7 illustrates some data taken during such a calibration to determine EHT strain as a function of applied scaffold strain. For this sample, the EHT strain is approximately $86 \%$ of the scaffold strain through the entire range of actuation. Each EHT sample in all experiments reported here was evaluated to assess this strain mapping relationship.

For uniaxial loading on a linear elastic tissue, the mechanical definition of tissue strain is:

$$
\varepsilon_{t}=\frac{\Delta L_{c}}{L_{c}}
$$

where $\boldsymbol{L}_{\boldsymbol{c}}$ defines the span of the of central region of the EHT over which strain is to be measured (as indicated in Fig. 7). In our experiments, image feature extraction software (MATLAB) was used to process images acquired during scaffold stretching to evaluate changes in strain.

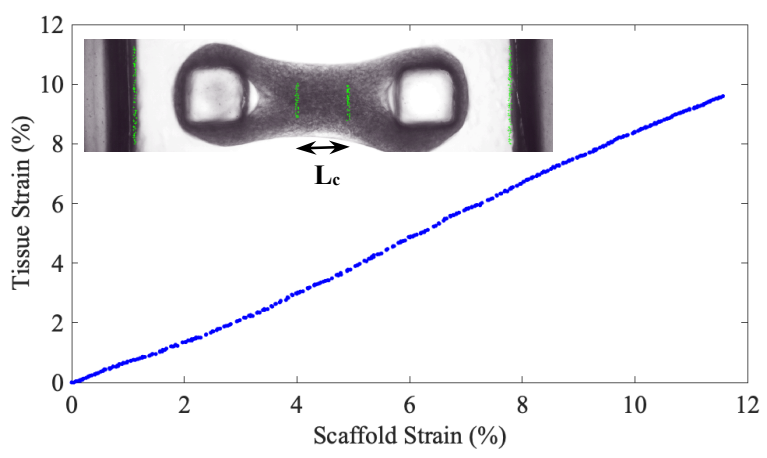

Fig. 7. Measured strain of an EHT sample as a function of applied scaffold strain. The scaffold and tissue strains are measured relative to the reference length of a central region of the EHT (Lc) and the reference well-edge separation when the scaffold is unstrained. These reference lengths are estimated using a point tracking algorithm in MATLAB. Tracked points are shown in the inset photo as green markers. As the scaffold is stretched by the actuators, the measured tissue strain varies in proportion to the scaffold strain, with a proportionality constant of $\sim 0.86$ for this EHT. The magnitude of imposed strain measured in the central region of the EHT depends on the geometry of EHT attachments to the pillars, among other factors, but is always proportional to scaffold strain. For EHTs observed in this work that proportionality constant has ranged from 0.4 to 1.0. 


\section{E. Feedback Control System}

In the experiments reported here, integral feedback control was used to maintain the specified control objective. More complex control approaches, including those with proportional and derivative feedback and those with feedforward compensation might improve controller performance. Active electrical pacing of the tissue might also improve controller performance by making the onset of contractile forces more predictable temporally.

To determine if the measured system closed loop control bandwidth is adequate to regulate force in a spontaneously contracting EHT, we first estimated the amplitude spectrum of the time series of recorded spontaneous contractions (depicted in Fig. 5) over a bandwidth of $\sim 156 \mathrm{~Hz}$ (the Nyquist limit for our $312 \mathrm{~Hz}$ image acquisition rate). The measured amplitude spectrum from $0-20 \mathrm{~Hz}$ is shown in Figure 6. The measured signal is indistinguishable from noise for all frequencies above $10 \mathrm{~Hz}$.

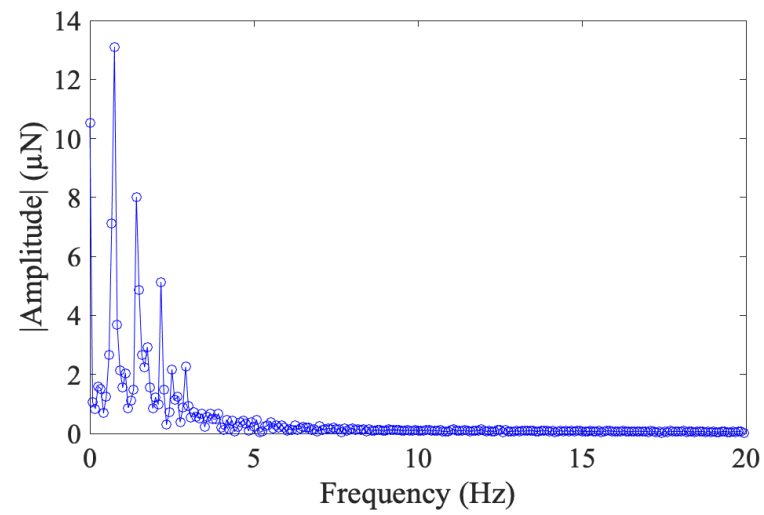

Fig. 6. EHT amplitude response as a function of frequency for the spontaneous EHT contraction data depicted in Figure 5. The measurement system has a temporal bandwidth of $\sim 156 \mathrm{~Hz}$, and an estimated noise floor of $\sim 0.5 \mu \mathrm{N}$. The signal is indistinguishable from noise for frequencies above $10 \mathrm{~Hz}$.

Next, we evaluated the system closed-loop control frequency response in an experiment in which the control loop was implemented on a $\mu$ Tug without tissue. The control objective was to produce an instantaneous small step change in the scaffold strain $(2.5 \%)$ corresponding to about one fifth of the achievable control range of achievable strain. Strain response of the scaffold was recorded in real time. The time constant associated with the strain step response was found to be $0.0144 \mathrm{~s}$, with a standard deviation of $0.0024 \mathrm{~s}$. The time constant corresponds to the time needed for the strain to reach $63 \%$ of the way from the commanded initial state to the commanded final state in response to a step change in input. This definition of time constant assumes a first order linear response and ignores nonlinearities and hysteresis of the piezoelectric actuator but provides a reasonable estimate for bandwidth calculation. The estimated time constant implies a small signal bandwidth of $69 \mathrm{~Hz}$ for the closed-loop control system.

We conclude that the system has a sufficient bandwidth to control all the measurable dynamics associated with spontaneous EHTs contractile forces in $\mu$ Tugs.

\section{RESULTS}

We conducted experiments in which we used force control feedback to enforce isometric boundary conditions for the EHT. In all experiments, the measured spontaneous peak contractile force under isometric control increased in comparison to the measured spontaneous peak contractile force under auxotonic conditions for the same tissue.

Since the force control system uses pillar displacement as its feedback signal, some deflection of that pillar must occur before the actuators can counteract that motion. Hence the boundary stiffness is not infinite but is limited by the increment of pillar deflection required to generate a feedback signal. We estimated that under closed loop control the effective stiffness of the scaffold is $>250 \mathrm{~N} / \mathrm{m}$, at least fifty times stiffer than without control.

Closed-loop control experiments were performed on EHTs with a control objective to maintain isometric boundary conditions dynamically for tissues under several different preload conditions, with tissue static strain, measured relative to the tissue in an unstrained scaffold, ranging from $1.3 \%$ to $16 \%$. in comparison to its original length (nominally $\sim 1.6 \mathrm{~mm}$ ). The EHT was subject to closed loop control for 75 seconds in each experiment, with 10 seconds of pre-control and 10 seconds of post-control monitoring of tissue force under auxotonic conditions.

Figure 8 illustrates in a typical experimental result showing the effectiveness of the control algorithm in maintaining fixed EHT length despite spontaneous contractile force perturbations. Figure 9 is a plot of the EHT forces measured for the experimental result depicted in Figure 8. Prior to control, the EHT auxotonic peak contractile force was measured to be about $66 \mu \mathrm{N}$. When controlled, the EHT isometric peak contractile force increased to $\sim 124 \mu \mathrm{N}$, nearly twice as large as the auxotonic peak contractile force. Force during control was calculated as described previously in Eq. 1.

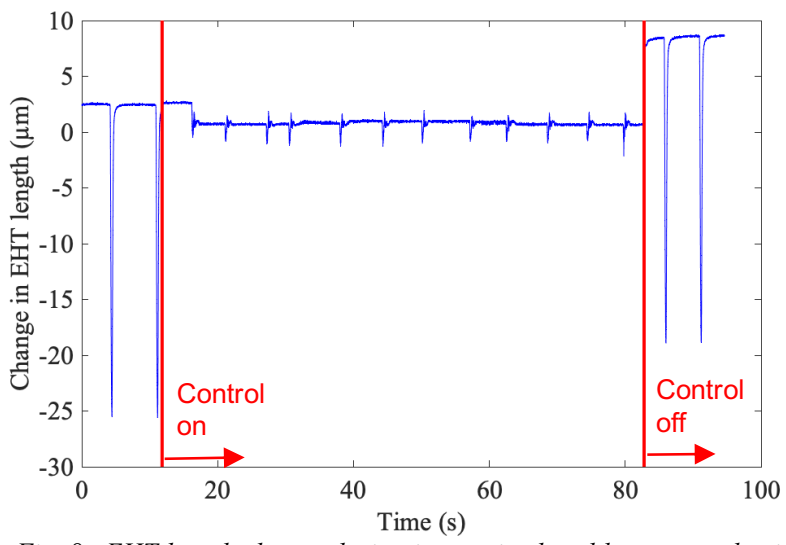

Fig. 8. EHT length change during isometric closed loop control, with the control objective to hold tissue at fixed length irrespective of contractile force. Tissue nominal strain is $1.8 \%$ with respect to initial EHT state. In the ten seconds prior to initiating control, the EHT contractile force periodically deforms the pillars resulting in tissue contraction. Upon initiation of control the pillars are maintained at a nominally fixed separation through active feedback to the $\mu$ Tug via piezoelectric actuation. 
We conducted experiments on four EHTs in which we first applied a static preload to produce strain in the EHT, (using the PZT actuator to statically stretch the scaffold) and then implemented control to monitor the ratio of isometric EHT contractile force to auxotonic EHT contractile force. The results are summarized in Figure 10.

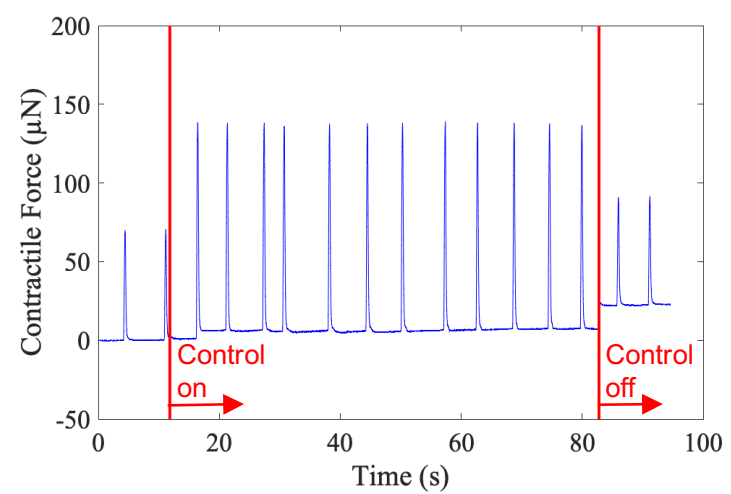

Fig. 9. EHT contractile force measured during a typical isometric control experiment. The peak-to-valley magnitude of spontaneous contractile force increased by a factor of 1.88 during isometric boundary control in comparison to spontaneous contractile force without control.

Preload EHT strains ranged from $1.2 \%$ to $12.9 \%$. The mean ratio of peak contractile force for controlled (isometric) and uncontrolled (auxotonic) constraints was 1.78 with a standard deviation of 0.15 .

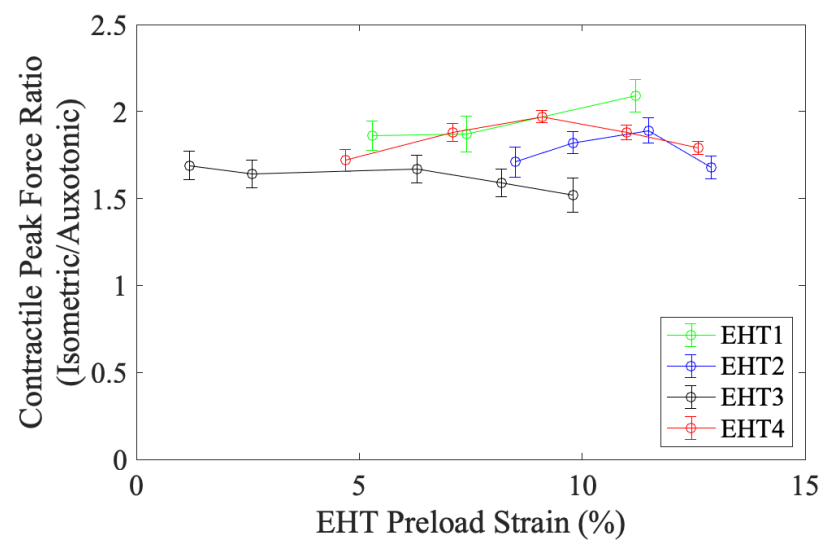

Fig. 10. Ratio of peak contractile force for isometric control to peak contractile force for auxotonic control measured for four different EHTs, with each EHT subjected to several different preload strains ranging from $1.2 \%$ to 12.6\%. Circles represent recorded data. Each tissue state was measured only once. Error bars correspond to the range of expected errors assuming measurement noise of $2.5 \mu \mathrm{N}$ for all force measurements. Colors correspond to different tissues. All EHTs exerted forces increase by 50-100\% in response to being subjected to isometric boundary conditions. No significant influence of preload on peak contractile force ratio was observed over the range of conditions tested.

Errors in pillar stiffness measurement affect both controlled and uncontrolled contractile force measurement equally, so those errors will not affect the ratio calculation. Since only pillar top separation and well separation are measured during control, systematic errors in estimates in pillar bottom separation needed for peak force calculation could affect the calculated force ratio. Since pillar bottom separation estimates are based indirectly on measurements of well edge separation, errors in that mapping need to be evaluated.

We conducted two experiments to try to evaluate and limit that potential source of error. First, we actuated scaffolds with no EHT over $13 \%$ scaffold strain range while inferring contractile force from pillar top separation and well separation measurements. The inferred pillar bending force should remain zero in this case since there is no tissue to exert a load. We found that the inferred force remained below $10 \mu \mathrm{N}$ for the entire range of scaffold strain and below $2 \mu \mathrm{N}$ over the range of scaffold strains typically associated with control experiments $(2.5 \%)$. As described previously, we also validated the correlation between pillar bottom separation and well edge separation in quasistatic experiments with scaffolds containing EHTs. In these experiments we employed dual cameras, one focused on the well edges and one focused on pillar bottoms to measure their separations simultaneously. The results showed high fidelity in the presumed linear relationship between well edge separation and pillar bottom separation.

\section{Discussion}

Values of peak contractile force before (auxotonic) and during (isometric) control, along with peak contractile force ratio and preload tissue strain and for the dataset plotted in Figure 10 are shown in Table 1. The results point to a strong correlation between effective boundary stiffness, with a force ratio between 1.5 and 2.1. This ratio appears to be uncorrelated with the spontaneous peak contractile force amplitude for EHTs, which ranged from $32.4 \mu \mathrm{N}$ to $104.9 \mu \mathrm{N}$ for the samples tested. The ratio also appears to be uncorrelated with preload strain over the range of preload strains tested, ranging from $1.2 \%$ to $12.9 \%$.

Table 1. Peak force data for contraction of EHTs in Fig. 10

\begin{tabular}{l|c|c|c|c} 
Sample & $\begin{array}{c}\text { Auxotonic } \\
\text { peak force } \\
(\boldsymbol{\mu N})\end{array}$ & $\begin{array}{c}\text { Isometric } \\
\text { peak force } \\
(\boldsymbol{\mu N})\end{array}$ & $\begin{array}{c}\text { Force } \\
\text { ratio }\end{array}$ & $\begin{array}{c}\text { Tissue } \\
\text { strain } \\
(\%)\end{array}$ \\
\hline EHT1 & 42.3 & 78.6 & 1.9 & 5.3 \\
\hline EHT1 & 35 & 65.5 & 1.9 & 7.4 \\
\hline EHT1 & 42.3 & 88.4 & 2.1 & 11.2 \\
\hline EHT2 & 39.2 & 67 & 1.7 & 8.5 \\
\hline EHT2 & 56.2 & 102.2 & 1.8 & 9.8 \\
\hline EHT2 & 49.5 & 93.6 & 1.9 & 11.5 \\
\hline EHT2 & 51.3 & 86.2 & 1.7 & 12.9 \\
\hline EHT3 & 40.7 & 68.8 & 1.7 & 1.2 \\
\hline EHT3 & 41.7 & 68.4 & 1.6 & 2.6 \\
\hline EHT3 & 42.7 & 71.4 & 1.7 & 6.3 \\
\hline EHT3 & 40.4 & 64.2 & 1.6 & 8.2 \\
\hline EHT3 & 32.4 & 49.3 & 1.5 & 9.8 \\
\hline EHT4 & 53.5 & 92 & 1.7 & 4.7 \\
\hline EHT4 & 70 & 131.7 & 1.9 & 7.1 \\
\hline EHT4 & 104.9 & 206.6 & 2.0 & 9.1 \\
\hline EHT4 & 87.7 & 164.9 & 1.9 & 11 \\
\hline EHT4 & 91.8 & 164.3 & 1.8 & 12.6 \\
\hline
\end{tabular}


In one extended-duration experiment, we maintained isometric control for a duration of fifteen minutes. The isometrically controlled peak contractile force increased immediately with respect to the pre-control peak contractile force by a factor of 1.8 (from $16 \mu \mathrm{N}$ to $28 \mu \mathrm{N}$ ). Over the course of fifteen minutes of isometric control, the isometrically controlled peak contractile force increased by about $14 \%$ from its initial value (from $28 \mu \mathrm{N}$ to $32 \mu \mathrm{N}$ ). After control was stopped, the peak contractile force declined from $32 \mu \mathrm{N}$ to $18 \mu \mathrm{N}$, again corresponding to a change in contractile peak force ratio of 1.8 at the transition. The result is illustrated in Figure 11.

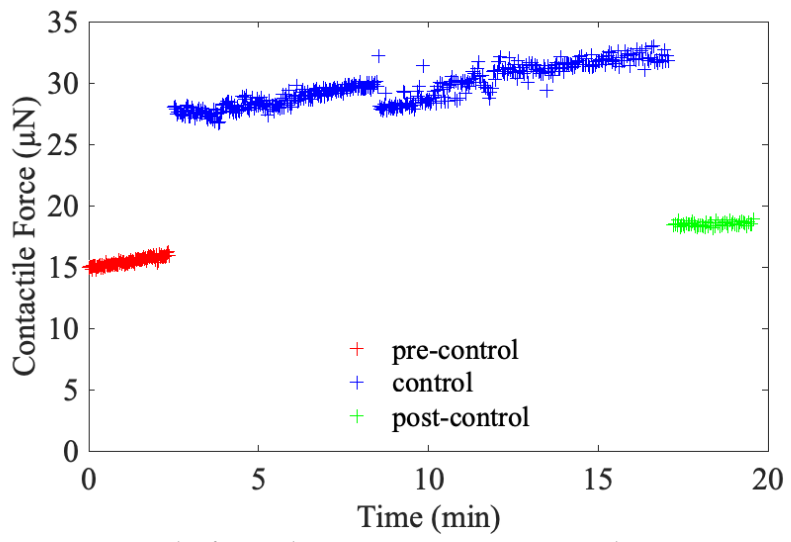

Fig. 11. Results from a longer-term isometric control experiment in which control was maintained for fifteen minutes while monitoring peak contractile forces on an EHT. The ratio of peak contractile forces during control to those just before and just after the control was about 1.8.

The system described here can be used for EHT force control experiments other than increasing the effective stiffness of scaffold constraints. With open loop bandwidth of $>150 \mathrm{~Hz}$, the actuation system is capable of imposing arbitrary dynamic tensile forces on the EHT with peak forces up to an order of magnitude larger than the typical contractile force that the EHT produces. This capacity enables mechanical conditioning, with or without direct feedback. Inspired by recent results that indicate that long-term open-loop electrical pacing at variable rates can improve EHT maturation, we could use this apparatus with or without electrical pacing to explore whether mechanical conditioning could have a similar impact on EHT maturation.

We have adapted the apparatus, adding a second camera focused on the EHT, to allow real time stiffness measurement of engineered cardiac tissues. For that purpose, the piezoelectric actuators stretch the scaffold and tissue while the imaging system records strain and infers contractile force and EHT tensile stress. Because of the high bandwidth of the system, this stiffness measurement can be done at low or high strain rates to characterize viscoelastic properties of EHT.

\section{CONCLUSION}

We have described a novel EHT force control system that has the capacity to alter preload and afterload of EHTs and to regulate scaffold stiffness through direct force feedback. The imposition of rigid boundary constraints on EHTs has been shown to reliably increase the contractile force exerted by the EHT by $50-100 \%$ in comparison to auxotonic constraints.

\section{ACKNOWLEDGMENT}

The authors would like to thank P. Cloonan for assistance with stem cell culture.

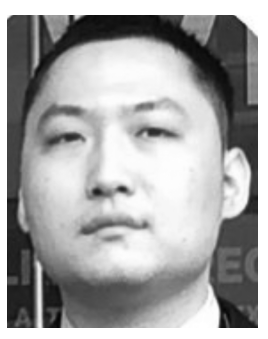

\section{Biographies}

Huate Li received a B.S. degree in mechanical engineering from Shanghai Jiao Tong University, Shanghai, PRC, in 2015, and an M.S. in mechanical engineering from Columbia University in New York, New York in 2017. He is currently a Ph.D. candidate in mechanical engineering at Boston University, Boston, MA int the Precision Engineering Research Laboratory at the Boston University Photonics Center. His research interests include tissue engineering, optical microscopy, adaptive optics, and real-time control system development.

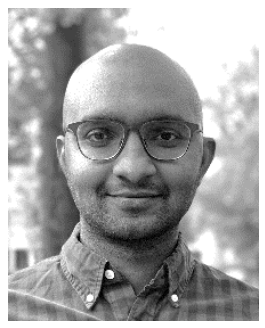

Subramanian Sundaram received the B.E.(Hons.) degrees in electrical engineering and mechanical engineering from the Birla Institute of Technology and Science (BITS), Pilani, India, in 2010, and the S.M. and Ph.D. degrees in electrical engineering and computer science from the Massachusetts Institute of Technology (MIT), Cambridge, MA in 2014 and 2018 respectively. He is currently an American Heart Association Postdoctoral Fellow at Boston University and the Wyss institute for biologically inspired engineering, Harvard University. His research interests include robotics, advanced fabrication, and tissue engineering.

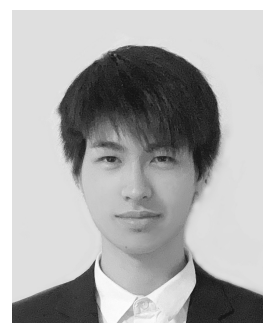

Ruifeng Hu received a B.S. degree in mechanical engineering from University of Pittsburgh, Pittsburgh, Pennsylvania, in 2020. From 2018-2019, he worked as an undergraduate researcher in Center for Robotics and Automation, Department of Biomedical engineering, City University of Hongkong, Hongkong, PRC. He is currently a Ph.D. student in mechanical engineering at Boston University, Boston, MA. His research interests include bio-imaging, tissue engineering, cardiac electrophysiology, closed-loop cells manipulation,

and optical tweezers.

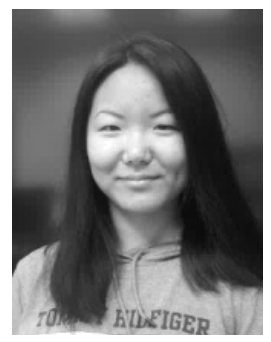

Lihua Lou completed her Ph.D. degree in Environmental Toxicology in 2019 from Texas Tech University under the supervision of Prof. Seshadri Ramkumar. Lihua is currently working in a postdoctoral role in Prof. Arvind Agarwal's laboratory at the Mechanical and Materials Department, Florida International University. Her research focuses on measuring nano-mechanics of soft biological materials (e.g., engineered cardiac tissues, porcine myocardium, biomedical scaffolds, hydrogels, and nanofibers) as well as designing and application of functional nanomaterials. She is a recipient of multiple awards and scholarships from Phi Kappa Phi, AATCC, Terracon Funding, and Texas Tech University. 


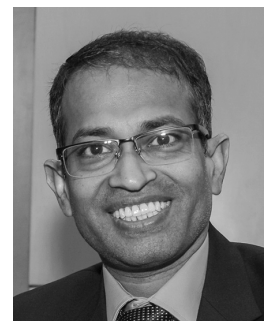

Arvind Agarwal is the Chairman and a Professor in the Department of Mechanical and Materials Engineering at the Florida International University. $\mathrm{He}$ received his Ph.D. in Materials Science and Engineering from the University of Tennessee, Knoxville in 1999 and B. Tech. and M. Tech. from Indian Institute of Technology (IIT), Kanpur in 1993 and 1995, respectively. His current research interests include graphene and Boron Nitride nanotube (BNNT) reinforced composites and coatings, thermal spray, surface engineering, spark plasma sintering (SPS), nano mechanics and nanotribology, biomaterials and nano mechanics of cells and tissues. He has published 320 technical articles, including 251 peer-reviewed journal articles, 3 books, and 7 edited books.

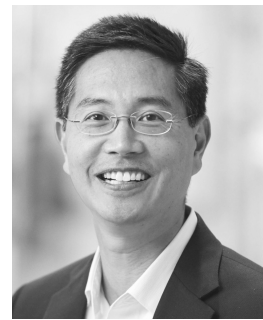

Chris Chen is the William Fairfield Warren Distinguished Professor at Boston University, director of the Tissue Microfabrication Laboratory, and founding director of the Biological Design Center at Boston University. He received his A.B. in Biochemistry from Harvard, M.S. in Mechanical Engineering from MIT, and Ph.D. in Medical Engineering and Medical Physics from the HarvardMIT. Health Sciences and Technology Program. He earned his M.D. from the Harvard Medical School. He has been an instrumental figure in the development of engineered cellular microenvironments to understand and control how cells build tissues. He has received numerous honors, including the Presidential Early Career Award for Scientists and Engineers, the Angiogenesis Foundation Fellowship, the Office of Naval Research Young Investigator Award, the Mary Hulman George Award for Biomedical Research, the Herbert W. Dickerman Award for Outstanding Contribution to Science, and the Robert A. Pritzker Award. He serves a Fellow of the American Institute for Medical and Biological Engineering, and as a member of the Faculty of 1000 Biology, the Board of Trustees for the Society for BioMEMS and Biomedical Nanotechnology, and Defense Sciences Study Group.

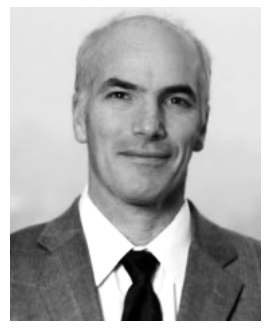

Thomas Bifano received B.S. (1980) and M.S. (1983) degrees in mechanical engineering and materials science from Duke University and a Ph.D. degree (1988) in mechanical engineering from North Carolina State University. He is a Professor in the Mechanical Engineering Department, Boston University, Boston, MA, with joint appointments in the Biomedical Engineering Department, the Electrical and Computer Engineering Department, and the Materials Science Division. He directs the Boston University Photonics Center. His research interests include precision engineering, adaptive optics, microscopy, and tissue mechanics. He is co-founder and CTO of Boston Micromachines Corporation, Cambridge, MA.

\section{REFERENCES}

[1] G. Campostrini, L. M. Windt, B. J. van Meer, M. Bellin, and C. L. Mummery, "Cardiac Tissues From Stem Cells: New Routes to Maturation and Cardiac Regeneration," (in eng), Circ Res, vol. 128, no. 6, pp. 775-801, Mar 19 2021, doi: 10.1161/circresaha.121.318183.

[2] J. T. Hinson, A. Chopra, N. Nafissi, W. J. Polacheck, C. C. Benson, S. Swist, J. Gorham, L. Yang, S. Schafer, C. C. Sheng, A. Haghighi, J. Homsy, N. Hubner, G. Church, S. A. Cook, W. A. Linke, C. S. Chen, J. G. Seidman, and C. E. Seidman, "Titin mutations in iPS cells define sarcomere insufficiency as a cause of dilated cardiomyopathy," Science, vol. 349, no. 6251, pp. 982-986, 2015, doi: 10.1126/science.aaa5458.

[3] B. M. Ogle, N. Bursac, I. Domian, N. F. Huang, P. Menasché, C. E. Murry, B. Pruitt, M. Radisic, J. C. Wu, S. M. Wu, J. Zhang, W. H. Zimmermann, and G. Vunjak-Novakovic, "Distilling complexity to advance cardiac tissue engineering," (in eng), Sci Transl Med, vol. 8, no. 342, p. 342ps13, Jun 8 2016, doi: 10.1126/scitranslmed.aad2304.

[4] W. R. Legant, A. Pathak, M. T. Yang, V. S. Deshpande, R. M. McMeeking, and C. S. Chen, "Microfabricated tissue gauges to measure and manipulate forces from 3D microtissues," Proceedings of the National Academy of Sciences, vol. 106, no. 25, pp. 10097-10102, 2009, doi: 10.1073/pnas.0900174106.

[5] M. N. Hirt, A. Hansen, and T. Eschenhagen, "Cardiac tissue engineering: state of the art," (in eng), Circ Res, vol. 114, no. 2, pp. 354-67, Jan 17 2014, doi: 10.1161/circresaha.114.300522.

[6] S. S. Nunes, J. W. Miklas, J. Liu, R. Aschar-Sobbi, Y. Xiao, B. Zhang, J. Jiang, S. Massé, M. Gagliardi, A. Hsieh, N. Thavandiran, M. A. Laflamme, K. Nanthakumar, G. J. Gross, P. H. Backx, G. Keller, and M. Radisic, "Biowire: a platform for maturation of human pluripotent stem cell-derived cardiomyocytes," Nat Methods, vol. 10, no. 8, pp. 781787, 2013/08/01 2013, doi: 10.1038/nmeth.2524.

J. Schwan and S. G. Campbell, "Prospects for In Vitro Myofilament Maturation in Stem Cell-Derived Cardiac Myocytes," (in eng), Biomark Insights, vol. 10, no. Suppl 1, pp. 91-103, 2015, doi: 10.4137/bmi.S23912.

A. Leonard, A. Bertero, J. D. Powers, K. M. Beussman, S. Bhandari, M. Regnier, C. E. Murry, and N. J. Sniadecki, "Afterload promotes maturation of human induced pluripotent stem cell derived cardiomyocytes in engineered heart tissues," (in eng), J Mol Cell Cardiol, vol. 118, pp. 147-158, 2018, doi: 10.1016/j.yjmcc.2018.03.016.

J.-L. Ruan, N. L. Tulloch, M. Saiget, S. L. Paige, M. V. Razumova, M. Regnier, K. C. Tung, G. Keller, L. Pabon, H. Reinecke, and C. E. Murry, "Mechanical Stress Promotes Maturation of Human Myocardium From Pluripotent Stem Cell-Derived Progenitors," (in eng), Stem Cells, vol. 33, no. 7, pp. 2148-2157, 2015, doi: 10.1002/stem.2036. 
[10] O. J. Abilez, E. Tzatzalos, H. Yang, M. T. Zhao, G. Jung, A. M. Zöllner, M. Tiburcy, J. Riegler, E. Matsa, P. Shukla, Y. Zhuge, T. Chour, V. C. Chen, P. W. Burridge, I. Karakikes, E. Kuhl, D. Bernstein, L. A. Couture, J. D. Gold, W. H. Zimmermann, and J. C. $\mathrm{Wu}$, "Passive Stretch Induces Structural and Functional Maturation of Engineered Heart Muscle as Predicted by Computational Modeling," (in eng), Stem Cells, vol. 36, no. 2, pp. 265-277, Feb 2018, doi: 10.1002/stem.2732.

[11] X. Yang, L. Pabon, and C. E. Murry, "Engineering adolescence: maturation of human pluripotent stem cell-derived cardiomyocytes," (in eng), Circulation research, vol. 114, no. 3, pp. 511-523, 2014, doi: 10.1161/CIRCRESAHA.114.300558.

[12] F. Xu, R. Zhao, A. S. Liu, T. Metz, Y. Shi, P. Bose, and D. H. Reich, "A microfabricated magnetic actuation device for mechanical conditioning of arrays of 3D microtissues," Lab Chip, 10.1039/C4LC01395F vol. 15, no. 11, pp. 2496-2503, 2015, doi: 10.1039/C4LC01395F.

[13] C. Fink, S. Ergün, D. Kralisch, U. Remmers, J. Weil, and T. Eschenhagen, "Chronic stretch of engineered heart tissue induces hypertrophy and functional improvement," (in eng), Faseb $J$, vol. 14, no. 5, pp. 669-79, Apr 2000, doi: 10.1096/fasebj.14.5.669.

[14] A. Mihic, J. Li, Y. Miyagi, M. Gagliardi, S.-H. Li, J. $\mathrm{Zu}, \mathrm{R}$. D. Weisel, G. Keller, and R.-K. Li, "The effect of cyclic stretch on maturation and $3 \mathrm{D}$ tissue formation of human embryonic stem cell-derived cardiomyocytes," Biomaterials, vol. 35, no. 9, pp. 2798-2808, 2014/03/01/ 2014, doi: https://doi.org/10.1016/j.biomaterials.2013.12.052.

[15] S.-J. Gwak, S. H. Bhang, I.-K. Kim, S.-S. Kim, S.-W. Cho, O. Jeon, K. J. Yoo, A. J. Putnam, and B.-S. Kim, "The effect of cyclic strain on embryonic stem cellderived cardiomyocytes," Biomaterials, vol. 29, no. 7, pp. $844-856, \quad 2008 / 03 / 01 / 2008$, doi: https://doi.org/10.1016/j.biomaterials.2007.10.050.

[16] I. Mannhardt, C. Warncke, H. K. Trieu, J. Müller, and T. Eschenhagen, "Piezo-bending actuators for isometric or auxotonic contraction analysis of engineered heart tissue," (in eng), J Tissue Eng Regen Med, vol. 13, no. 1, pp. 3-11, Jan 2019, doi: 10.1002/term.2755.

[17] A. K. Schroer, M. S. Shotwell, V. Y. Sidorov, J. P. Wikswo, and W. D. Merryman, "I-Wire Heart-on-aChip II: Biomechanical analysis of contractile, threedimensional cardiomyocyte tissue constructs," (in eng), Acta Biomater, vol. 48, pp. 79-87, Jan 15 2017, doi: 10.1016/j.actbio.2016.11.010.

[18] V. Y. Sidorov, P. C. Samson, T. N. Sidorova, J. M. Davidson, C. C. Lim, and J. P. Wikswo, "I-Wire Heart-on-a-Chip I: Three-dimensional cardiac tissue constructs for physiology and pharmacology," (in eng), Acta Biomater, vol. 48, pp. 68-78, Jan 15 2017, doi: 10.1016/j.actbio.2016.11.009.

X. Lian, J. Zhang, S. M. Azarin, K. Zhu, L. B. Hazeltine, X. Bao, C. Hsiao, T. J. Kamp, and S. P. Palecek, "Directed cardiomyocyte differentiation from human pluripotent stem cells by modulating Wnt/ $\beta$ catenin signaling under fully defined conditions," Nat Protoc, vol. 8, no. 1, pp. 162-175, 2013/01/01 2013, doi: $10.1038 /$ nprot.2012.150. 